\title{
Taxotere-induced elevated expression of IL8 in carcinoma-associated fibroblasts of breast invasive ductal cancer
}

\author{
YINGLIANG LI ${ }^{1,2}$, GUOHUA RONG ${ }^{3}$ and HUA KANG ${ }^{1}$ \\ ${ }^{1}$ Department of General Surgery, Xuanwu Hospital, Capital Medical University, Beijing 100053; \\ ${ }^{2}$ Department of General Surgery, The First Affiliated Hospital of Nanchang University, Nanchang, Jiangxi 330006; \\ ${ }^{3}$ Department of General Surgery, Qingdao Municipal Hospital, Qingdao, Shandong 266011, P.R. China
}

Received May 18, 2015; Accepted September 28, 2016

DOI: $10.3892 / \mathrm{ol} .2017 .5612$

\begin{abstract}
Breast cancer is the most common malignant tumor in women worldwide, and accounts for an estimated $29 \%$ of new cases and $15 \%$ of cancer-associated mortalities each year. Invasive ductal carcinoma represents $70-80 \%$ of all breast cancer cases, which are responsible for the majority of breast cancer fatalities. Though great progress has been made in understanding the tumorigenesis and development of breast cancer, problems surrounding treatment persist. It was previously reported that carcinoma-associated fibroblasts (CAFs) may be closely associated with chemotherapy resistance. In the present study, primary-cultured CAFs from surgically resected breast invasive ductal cancer tissues were prepared and tested to clarify the change of gene expression profile following treatment with $20 \mathrm{ng} / \mathrm{ml}$ Taxotere ${ }^{\circledR}$ for $24 \mathrm{~h}$ through microarray analysis. In addition, quantitative polymerase chain reaction and western blotting were performed to compare the gene and protein expression of the candidate gene in CAFs prior to and following Taxotere treatment. Based on the obtained data, 35 differentially expressed genes were identified, including ACTA2, ACTC1, ACTG, ALDH1B1, AMY1A, C5orf13, CNN1, CXCR7, DDAH1, FGF1, PDLIM3, MAMLD1, MYH11, OXTR, PDLIM5, RARRES1, SERPINA3, TRIL, C14orf43, C1orf51, CXCL12, CXCL2, EGR2, EGR3, IER3, interleukin (IL)8, IRF1, JUNB, MMP1, NAV2, NFKBIA, NFKBIZ, TRIB1, WNT16 and ZC3H12A. It was observed that the expression of the candidate gene IL8 in the CAFs of breast invasive cancer following treatment with Taxotere was increased $(\mathrm{P}<0.05)$. Overall, elevated expression of IL8 induced by Taxotere in CAFs potentially supports the association between IL8 and chemotherapy response.
\end{abstract}

Correspondence to: Dr Hua Kang, Department of General Surgery, Xuanwu Hospital, Capital Medical University, 45 Changchun Road, Beijing 100053, P.R. China

E-mail: qiancong1984@163.com

Key words: carcinoma-associated fibroblasts, breast cancer, chemotherapy resistance, Taxotere, primary culture

\section{Introduction}

Breast cancer is one of the most common malignant tumors in women worldwide and accounts for an estimated $29 \%$ of new cases and $15 \%$ of cancer-associated mortalities each year $(1,2)$. Invasive ductal carcinoma represents $70-80 \%$ of all breast cancer cases, which are responsible for the majority of breast cancer fatalities. Though great progress has been made in understanding the tumorigenesis and development of breast cancer, problems surrounding treatment of these disease persist (3). As an important approach for breast cancer treatment, chemotherapy and neoadjuvant chemotherapy have already been challenged by drug resistance, even multi-drug resistance, with the underlying mechanisms remaining unclear (4). It was previously reported that the tumor microenvironment may be closely associated with chemotherapy resistance, which warrants further study $(5,6)$.

It is assumed that carcinoma-associated fibroblasts (CAFs), as major components of the tumor microenvironment, may be affected by chemotherapy treatment, resulting in an induced gene expression profile change. Furthermore, the differentially expressed genes in CAFs following chemotherapy may be important in modulating the function of tumor cells and participate in chemotherapy response correspondingly. Hence, the present study performed two steps. Firstly, the primary-cultured CAFs from surgically resected breast invasive ductal cancer tissues were prepared and examined to clarify the gene expression profile change following treatment with Taxotere ${ }^{\circledR}$, which has been commonly used in combined or single application chemotherapy regimens. Secondly, one differentially expressed gene was selected as the candidate gene for further analysis. The overall purpose was to investigate the effect of chemotherapy treatment on CAFs and determine the expression change of the candidate gene following chemotherapy, which may aid the understanding of the mechanism of chemotherapy resistance in breast cancer.

\section{Materials and methods}

Case selection. All tissues for primary cultures of CAFs were collected from 6 patients with breast invasive ductal cancer, who underwent surgical resection at Xuanwu Hospital, Capital Medical University (Beijing, China) between February 2014 
and July 2014. Only tissues in excess of those required for clinic diagnoses were harvested for the study. All patients were females ranging from 35-67 years (median, 49.3 years), and none had been treated with chemotherapy prior to surgery. Clinicopathological information for each patient was collected through a review of medical charts and pathology reports. The study was approved by the Institutional Review Board and Human Ethics Committee of Xuanwu Hospital, Capital Medical University. Written informed consent for using the samples for research purposes was obtained from all patients prior to surgery.

Cell cultures of CAFs and breast cancer cell line. Harvested tissues for primary cell culture of CAFs were placed in Dulbecco's modified Eagle's medium (DMEM) supplemented with $10 \%$ fetal bovine serum (FBS) and antibiotics (penicillin and streptomycin; Invitrogen; Thermo Fisher Scientific, Inc., Waltham, MA, USA) for immediate transportation on ice to the laboratory. Tissues were minced into small pieces, washed with PBS three times and digested for $20 \mathrm{~h}$ at $37^{\circ} \mathrm{C}$ in prepared reagent containing collagenase type I and hyaluronidase (Invitrogen; Thermo Fisher Scientific, Inc.). The cell suspension was filtrated using a 100 mesh screen and centrifuged at $167.85 \mathrm{x} g$ for $5 \mathrm{~min}$; the pellet was subsequently resuspended in the fresh DMEM containing 10\% FBS. Cell counting was performed with the $Z^{\mathrm{TM}}$ Series COULTER COUNTER ${ }^{\circledR}$ Cell and Particle Counter (Beckman Coulter, Inc., Brea, CA, USA). The population doubling was estimated based on the increase in cell number counted at each passage time. Furthermore, MDA-MB-231 cells (Sigma-Aldrich; Merck Millipore, Darmstadt, Germany) were cultured in DMEM supplemented with $10 \%$ FBS as the breast cancer cell line, according to the normal procedure.

$m R N A$ expression profiling. A total of 6 pairs of CAFs were prepared for microarray analysis. Each pair was obtained from the same patient and classified into two groups. One group was treated with $20 \mathrm{ng} / \mathrm{ml}$ Taxotere for $24 \mathrm{~h}$ (regarded as post-chemotherapy), while the other group was not treated with Taxotere (regarded as pre-chemotherapy). Total RNA was extracted from all cultured CAFs using the RNeasy ${ }^{\circledR}$ Mini kit (Qiagen, Inc., Valencia, CA, USA) according to the manufacturer's protocol. Microarray studies were performed at the Capital Medical University Microarray Centre, using a HumanHT-12 v4 Expression BeadChip kit (Illumina, Inc., San Diego, CA, USA) based on the BeadStation 500G system (Illumina, Inc.). The biotinylated cRNA preparation, hybridization and scanning of microarrays were performed according to the manufacturer's protocols. Biological replicates were used to reduce errors. The Gene Expression BeadChips have internal control features to monitor data quality. GenomeStudio software version 2.3.47 (Illumina, Inc.) was used to calculate and report $\mathrm{P}$-values, which represents the confidence that a given transcript is expressed above the background defined by negative control probes. A detection P-value $<0.01$ indicated that a gene could be considered as expressed. Differentially expressed genes in the CAFs pre-chemotherapy vs. post-chemotherapy were also identified as candidate genes and analyzed using the GenomeStudio software. The output was filtered to include genes whose expression was altered at least two-fold.
Reverse transcription-quantitative polymerase chain reaction (RT-qPCR). RT-qPCR was performed to confirm differential expression of the candidate gene interleukin (IL) 8 in cultured CAFs pre- and post-chemotherapy (treated with $20 \mathrm{ng} / \mathrm{ml}$ Taxotere for $24 \mathrm{~h}$ ), using an iQ5 Real-Time PCR Detection system (Bio-Rad Laboratories, Inc., Hercules, CA, USA). cDNA was synthesized using $1 \mu \mathrm{g}$ total RNA, oligo(dT) and Superscript $^{\mathrm{TM}}$ III Reverse Transcriptase (Invitrogen; Thermo Fisher Scientific, Inc.). Synthesis was performed according to the manufacturer's protocol. All primers were designed using Primer Express software version 3.0.1 (Applied Biosystems; Thermo Fisher Scientific, Inc.) for IL8 (upstream, ATACTC CAAACCTTTCCACCC and downstream, TCTGCACCC AGTTTTCCTTG). $\beta$-actin was used as a reference (upstream, CTCCATCCTGGCCTCGCTGT and downtstream, GCTGTC ACCTTCACCGTTCC) Predicted PCR product sequences were verified using the Basic Local Alignment Search Tool (https://blast.ncbi.nlm.nih.gov/Blast.cgi) for recognition of target and non-target sequences.

Western blot analysis. Western blotting was performed to evaluate the protein expression change of IL8 in CAFs pre- and post-chemotherapy. Briefly, the cells were lysed in radioimmunoprecipitation assay lysis buffer (1\% Nonidet $\mathrm{P}-40,1 \% \mathrm{w} / \mathrm{v}$ sodium deoxycholate, $0.1 \%$ sodium dodecyl sulfate, $0.15 \mathrm{M} \mathrm{NaCl}, 0.01 \mathrm{M}$ sodium phosphate and $2 \mathrm{mM}$ ethylenediaminetetraacetic acid), containing protease inhibitor cocktail (1 $\mathrm{mM}$ phenylmethanesulfonyl fluoride, $1 \mathrm{mM}$ protein inhibitors mixture and $1 \mathrm{mM}$ dithiothreitol). Proteins were separated by $7.5 \%$ SDS-PAGE, and were subsequently transferred to a nitrocellulose membrane and blocked in 5\% non-fat milk in washing buffer [10 mM Tris (pH, 7.5), $100 \mathrm{mM}$ $\mathrm{NaCl}$ and $0.1 \% \mathrm{w} / \mathrm{v}$ Tween 20] for $30 \mathrm{~min}$. Mouse anti-human IL8 monoclonal antibody (dilution, 1:1,000; catalogue no. ab10768; Abcam, Cambridge, MA, USA) and incubated at room temperature for $1 \mathrm{~h}$, followed by incubation for $1 \mathrm{~h}$ at room temperature with the goat anti-mouse secondary antibody (dilution, 1:5,000; Santa Cruz Biotechnology, Inc, Dallas, TX, USA). ECL Advance Western Blotting Detection kit (GE Healthcare Bio-Sciences, Pittsburgh, PA, USA) was used for color development. Densitometry analysis was performed by using Quantity One 1-D Analysis software version 4.62 (Bio-Rad Laboratories, Inc.).

Statistical analysis. Statistical analysis was performed using SPSS v17.0 (SPSS, Inc., Chicago, IL, USA). All values are represented as the mean \pm standard deviation. Student's $t$-test was used to compare IL8 mRNA expression levels in samples pre-chemotherapy vs. samples post-chemotherapy. $\mathrm{P}<0.05$ was considered to indicate a statistically significant difference.

\section{Results}

Morphological change of CAFs in breast invasive cancer following treatment with Taxotere. The primary-cultured CAFs from surgically resected breast invasive ductal cancer tissues were morphologically characterized by a flat spindle shape, rich cytoplasm and flat ovoid nucleus (Fig. 1A). Following treatment with $20 \mathrm{ng} / \mathrm{ml}$ Taxotere for $24 \mathrm{~h}$, the CAFs demonstrated a degenerative change in morphology, including 
A

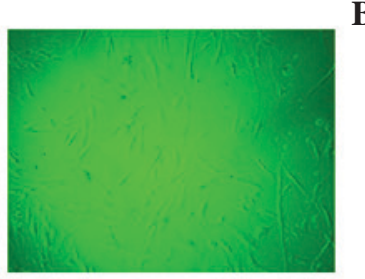

B

C

\begin{tabular}{|c|c|c|c|}
\hline Control & Treatment & $\begin{array}{l}\text { Gene } \\
\text { symbol }\end{array}$ & $\begin{array}{c}\text { Fold } \\
\text { change }\end{array}$ \\
\hline & & $\begin{array}{l}\text { EGR3 } \\
\text { C14orf } 43\end{array}$ & $\begin{array}{l}2.96 \\
2.41\end{array}$ \\
\hline & & NAV2 & 2.28 \\
\hline & & NFKBIA & 3.04 \\
\hline & & CXCL12 & 2.26 \\
\hline & & WNT16 & 4.85 \\
\hline & & TRIB1 & $\begin{array}{l}4.01 \\
3.35\end{array}$ \\
\hline & & NFKBI $Z$ & 3.03 \\
\hline & & 2C3H12A & 2.02 \\
\hline & & CXCL2 & 2.86 \\
\hline & & JUNB & 2.65 \\
\hline & & $\begin{array}{l}\text { IRF1 } \\
\text { lal }\end{array}$ & 2.45 \\
\hline & & IL8 & 2.38 \\
\hline
\end{tabular}

Figure 1. Effect of Taxotere treatment on CAFs in breast invasive ductal cancer tissue. (A) Characterized CAF morphology prior to treatment (magnification, x100). (B) Change in CAF morphology following treatment with $20 \mathrm{ng} / \mathrm{ml}$ Taxotere for $24 \mathrm{~h}$ (magnification, x100). (C) Represented hierarchical clustering of gene analysis expression of CAFs in breast invasive cancer pre- and post-chemotherapy (treated with Taxotere). CAF, carcinoma-associated fibroblast.

A

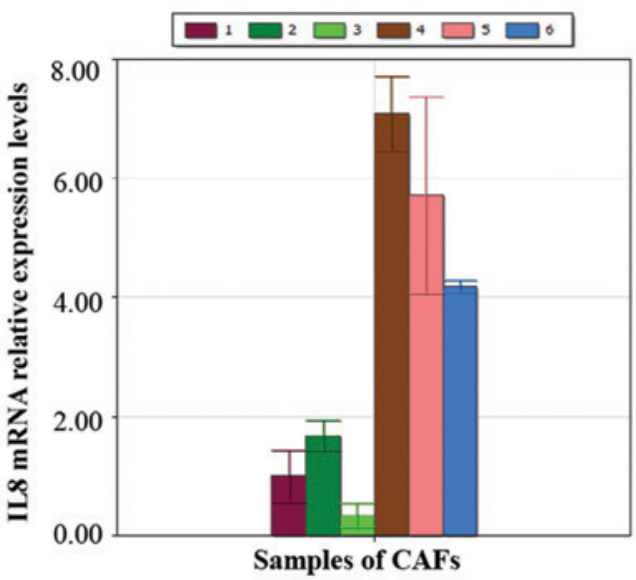

B

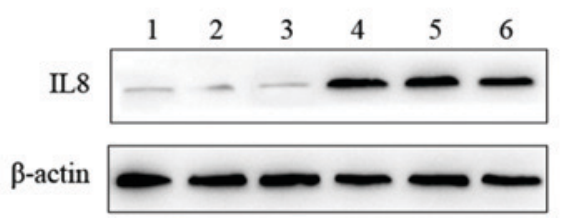

Figure 2. Enhanced gene expression of IL8 in the CAFs of breast invasive ductal cancer tissues following Taxotere treatment. (A) Elevated gene expression of IL8 in CAFs identified by reverse transcription-quantitative polymerase chain reaction. (D) Western blot representing the increased protein expression of IL8 following treatment with Taxotere. In (A) and (B), samples 1-3 were not treated with chemotherapy, while samples 4-6 were treated with Taxotere. IL8, interleukin 8; CAF, carcinoma-associated fibroblast.

an obscure shape, chromatic agglutination, karyopyknosis and nuclear fragmentation, which could be observed in a few of the cells under an inverted microscope (Fig. 1B).
Differentially expressed genes in the CAFs following treatment with Taxotere. Through mDNA expression profiling, 35 differentially expressed genes were identified, including ACTA2, ACTC1, ACTG, ALDH1B1, AMY1A, C5orf13, CNN1, CXCR7, DDAH1, FGF1, PDLIM3, MAMLD1, MYH11, OXTR, PDLIM5, RARRES1, SERPINA3, TRIL, C14orf43, C1orf51, CXCL12, CXCL2, EGR2, EGR3, IER3, IL8, IRF1, JUNB, MMP1, NAV2, NFKBIA, NFKBIZ, TRIB1, WNT16 and ZC3H12A. The candidate genes are represented in Fig. 1C. Based on the microarray data and literature retrieval, IL8, which has been reported to be correlated with carcinogenesis and development (7-9), was selected as the primary target for the following examinations.

Enhanced gene and protein expression of IL8 in the CAFs of breast cancer tissue following treatment with Taxotere. Using RT-qPCR, a higher expression of IL8 was observed in the CAFs following treatment with Taxotere compared with the control group pre-chemotherapy ( $\mathrm{P}=0.008$; Fig. $2 \mathrm{~A}$ ). These were results were also consistent with those obtained by western blot analysis (Fig. 2B).

\section{Discussion}

Breast cancer is the most common cancer among women worldwide (10). The disease places a considerable burden on patients and healthcare systems. Great progress has been made over the last few decades in terms of determining efficient approaches to treat breast cancer, however, problems regarding treatment persist. Drug resistance has severely restricted the application of chemotherapy and neoadjuvant chemotherapy, resulting in treatment failure. Therefore, various researchers have focused on the mechanism of chemotherapy resistance in breast cancer (11-13). Emerging evidences has indicated that the tumor microenvironment serves an important role in carcinogenesis and development, which has also been identified to participate in therapeutic resistance (14-16). CAFs have been considered as the most frequent components in tumor stroma (16), which thus warrants further investigation. It was reported that IL-17A was overexpressed by colorectal CAFs in response to chemotherapy, with expression validated directly in patient-derived specimens without culture, suggesting that chemotherapy promotes tumor microenvironment remodeling to support the tumor cellular hierarchy through secreted factors (5). Amornsupak et al (17) reported that pre-treatment of breast cancer cells with breast cancer-associated fibroblasts induced a degree of resistance to doxorubicin in accordance with the increased level of secreted high mobility group box 1 (HMGB1), highlighting the potential of stromal fibroblasts to contribute to chemoresistance in breast cancer cells partially through fibroblast-induced HMGB1 production (17).

Considering the close association between CAFs and chemotherapy resistance, it may be assumed that CAFs in breast cancer could be affected by chemotherapy treatment and exhibit an altered gene expression profile. Furthermore, the differentially expressed genes in CAFs following treatment with chemotherapy may be important in modulating the function of tumor cells, and subsequently participate in chemoresistance correspondingly. Based the present study, 


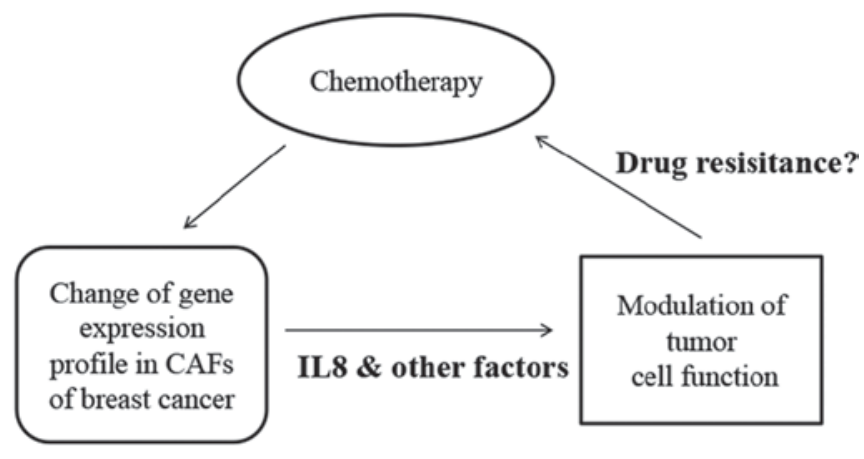

Figure 3. Proposed model presenting the inter-relationship between CAFs and chemotherapy treatment in breast cancer.

the primary-cultured CAFs from surgically resected breast invasive ductal cancer tissues were prepared and examined via BeadChip technology. It was demonstrated that the change in the gene expression profile occurred following treatment with Taxotere, which has been commonly used in combined or single application chemotherapy regimens. In total, 35 differentially expressed genes were identified, including ACTA2, ACTC1, ACTG, ALDH1B1, AMY1A, C5orf13, CNN1, CXCR7, DDAH1, FGF1, PDLIM3, MAMLD1, MYH11, OXTR, PDLIM5, RARRES1, SERPINA3, TRIL, C14orf43, C1orf51, CXCL12, CXCL2, EGR2, EGR3, IER3, IL8, IRF1, JUNB, MMP1, NAV2, NFKBIA, NFKBIZ, TRIB1, WNT16 and ZC3H12A. Among these candidate genes, IL8, which has been reported to have a close link with cancer development, was selected as the primary target for the study.

IL8, a multifunctional chemokine secreted by multiple cell types (including monocytes, endothelial cells, fibroblasts and tumor cells), has been frequently reported to be closely associated with cancer development and poor prognosis $(7,8,18)$. The exact role of IL8 in regulating tumor cell behavior remains unclear. It was reported that IL8 may participate in modulating tumor cell susceptibility and aggressiveness in breast cancer $(9,19)$. Furthermore, it was observed that elevated expression of IL8 by transfection increased the resistance to Adriamycin ${ }^{\circledR}$ in breast cancer cells (20). The aforementioned studies provided rationale for the current study to investigate the effect of IL8 on chemotherapy response in breast cancer. The present study observed higher expression of IL8 in CAFs of breast invasive cancer tissue following treatment with Taxotere compared with the control group pre-chemotherapy, thus indicating the link between IL8 and chemotherapy resistance. Considering the inter-relationship between CAFs and chemotherapy treatment, the present study proposes a model explaining chemoresistance in breast cancer, in which IL8 is involved (Fig. 3).

In conclusion, the present study demonstrated the change in the gene expression profile of CAFs of breast invasive cancer tissues pre- and post-treatment with Taxotere, and selected IL8 as the candidate gene. Taxotere-induced elevated expression of IL8 in CAFs supports the possible association between IL8 and chemotherapy response. The mechanism of chemoresistance in breast cancer requires further investigation in order to identify more efficient indicators for chemotherapy curative effect.

\section{Acknowledgements}

The present study was supported by the National Natural Science Fund of China (grant no. 81172517) and The Specialized Research Fund for the Doctoral Program of Higher Education (grant no. 20111107110001).

\section{References}

1. Siegel R, Ma J, Zou Z and Jemal A: Cancer statistics, 2014. CA Cancer J Clin 64: 9-29, 2014.

2. DeSantis C, Ma J, Bryan L and Jemal A: Breast cancer statistics, 2013. CA Cancer J Clin 64: 52-62, 2014.

3. Kim H, Jang SM, Ahn H, Sim J, Yi K, Chung Y, Han H, Rehman A, Chung MS, Jang K and Paik SS: Clinicopathological significance of dual-specificity protein phosphatase 4 expression in invasive ductal carcinoma of the breast. J Breast Cancer 18: 1-7, 2015.

4. Valero V, Vrdoljak E, Xu B, Thomas E, Gómez H, Manikhas A, Medina C, Li RK, Ro J, Bosserman L, et al: Maintenance of clinical efficacy after dose reduction of ixabepilone plus capecitabine in patients with anthracycline- and taxane-resistant metastatic breast cancer: A retrospective analysis of pooled data from 2 phase III randomized clinical trials. Clin Breast Cancer 12: 240-246, 2012.

5. Lotti F, Jarrar AM, Pai RK, Hitomi M, Lathia J, Mace A, Gantt GA Jr, Sukhdeo K, DeVecchio J, Vasanji A, et al: Chemotherapy activates cancer-associated fibroblasts to maintain colorectal cancer-initiating cells by IL-17A. J Exp Med 210: 2851-2872, 2013.

6. Tiago M, de Oliveira EM, Brohem CA, Pennacchi PC, Paes RD, Haga RB, Campa A, de Moraes Barros SB, Smalley KS and Maria-Engler SS: Fibroblasts protect melanoma cells from the cytotoxic effects of doxorubicin. Tissue Eng Part A 20: 2412-2421, 2014

7. Wang Y, Xu RC, Zhang XL, Niu XL, Qu Y, Li LZ and Meng XY: Interleukin-8 secretion by ovarian cancer cells increases anchorage-independent growth, proliferation, angiogenic potential, adhesion and invasion. Cytokine 59: 145-155, 2012.

8. Lee YS, Choi I, Ning Y, Kim NY, Khatchadourian V, Yang D, Chung HK, Choi D, LaBonte MJ, Ladner RD, et al: Interleukin-8 and its receptor CXCR2 in the tumour microenvironment promote colon cancer growth, progression and metastasis. Br J Cancer 106: 1833-1841, 2012.

9. Snoussi K, Mahfoudh W, Bouaouina N, Fekih M, Khairi H, Helal AN and Chouchane L: Combined effects of IL-8 and CXCR2 gene polymorphisms on breast cancer susceptibility and aggressiveness. BMC Cancer 10: 283, 2010.

10. Di Leo A, Curigliano G, Dieras V, Malorni L, Sotiriou C, Swanton C, Thompson A, Tutt A and Piccart M: New approaches for improving outcomes in breast cancer in Europe. Breast 24: 321-330, 2015.

11. Conley SJ, Baker TL, Burnett JP, Theisen RL, Lazarus D, Peters CG, Clouthier SG, Eliasof S and Wicha MS: CRLX101, an investigational camptothecin-containing nanoparticle-drug conjugate, targets cancer stem cells and impedes resistance to antiangiogenic therapy in mouse models of breast cancer. Breast Cancer Res Treat 150: 559-567, 2015.

12. Yao YS, Qiu WS, Yao RY, Zhang Q, Zhuang LK, Zhou F, Sun LB and Yue L: miR-141 confers docetaxel chemoresistance of breast cancer cells via regulation of EIF4E expression. Oncol Rep 33: 2504-2512, 2015.

13. Arfaoui A, Douik H, Kablouti G, Chaaben AB, Handiri N, Zid Z Ouni N, Zouiouch F, Ayari F, Mamoughli T, et al: Role of p53 Codon72 SNP in breast cancer risk and anthracycline resistance. Anticancer Res 35: 1763-1769, 2015.

14. Bezdenezhnykh N, Semesiuk N, Lykhova O, Zhylchuk V and Kudryavets Y: Impact of stromal cell components of tumor microenvironment on epithelial-mesenchymal transition in breast cancer cells. Exp Oncol 36: 72-78, 2014.

15. Mao Y, Keller ET, Garfield DH, Shen K and Wang J: Stromal cells in tumor microenvironment and breast cancer. Cancer Metastasis Rev 32: 303-315, 2013.

16. Fu Z, Song P, Li D, Yi C, Chen H, Ruan S, Shi Z, Xu W, Fu X and Zheng S: Cancer-associated fibroblasts from invasive breast cancer have an attenuated capacity to secrete collagens. Int J Oncol 45: 1479-1488, 2014. 
17. Amornsupak K, Insawang T, Thuwajit $\mathrm{P}$, O-Charoenrat $\mathrm{P}$, Eccles SA and Thuwajit C: Cancer-associated fibroblasts induce high mobility group box 1 and contribute to resistance to doxorubicin in breast cancer cells. BMC Cancer 14: 955, 2014.

18. Reis ST, Leite KR, Piovesan LF, Pontes-Junior J, Viana NI, Abe DK, Crippa A, Moura CM, Adonias SP, Srougi M and Dall'Oglio MF: Increased expression of MMP-9 and IL-8 are correlated with poor prognosis of bladder cancer. BMC Urol 12: 18,2012 .
19. Korantzis I, Kalogeras KT, Papaxoinis G, Kotoula V, Koutras A, Soupos N, Papakostas P, Dionysopoulos D, Samantas E, Christodoulou $\mathrm{C}$, et al: Expression of angiogenic markers in the peripheral blood of patients with advanced breast cancer treated with weekly docetaxel. Anticancer Res 32: 4569-4580, 2012.

20. Shi Z, Yang WM, Chen LP, Yang DH, Zhou Q, Zhu J, Chen JJ, Huang RC, Chen ZS and Huang RP: Enhanced chemosensitization in multidrug-resistant human breast cancer cells by inhibition of IL- 6 and IL-8 production. Breast Cancer Res Treat 135: 737-747, 2012. 